\title{
A Measure of Well-Spread Points in Noise Waves-Based Source Matrix for Wideband Noise Parameter Measurement: the SKA-Low Example
}

\author{
Adrian T. Sutinjo Senior Member, IEEE, Leonid Belostotski Senior Member, IEEE, Budi Juswardy Member, IEEE \\ and Daniel X. C. Ung Graduate Student Member, IEEE
}

\begin{abstract}
The existence of a figure of merit for measuring the degree of well-spread source points in noise parameter extraction has long been conjectured. This paper proposes a measure based on noise waves that is physically motivated and is directly connected to linear algebra through the matrix condition number and/or determinant. The key to this figure-of-merit is the selection of the noise temperature equation and the removal of singularity due to $1 /\left(1-\left|\Gamma_{s}\right|^{2}\right)$ factor. The result is a wellscaled source matrix with entries bounded within a unit circle. We demonstrate the effectiveness of this measure by extracting the noise parameters of an amplifier in the Low-Frequency Square Kilometre Array (SKA-Low) band of 50-350 MHz using 7 tuner positions. The noise parameters in the $50-100 \mathrm{MHz}$ band are successfully measured despite being below the $100 \mathrm{MHz}$ tuner rating. This outcome is very well predicted by the condition number and the determinant of the source matrix in question.
\end{abstract}

Index Terms-Low-noise amplifiers, noise measurements, thermal noise, UHF circuits, VHF circuits, radio astronomy.

\section{INTRODUCTION}

The Square Kilometre Array (SKA) is a next-generation radio telescope that will be hosted by South Africa and Australia. The Low-Frequency Square Kilometre Array (SKALow) telescope will be constructed in the Murchison Shire of Western Australia and will cover the $50-350 \mathrm{MHz}$ frequency band. A low-frequency radio telescope array may consist of hundreds to hundreds of thousands (e.g., SKA-Low) receiving antennas connected to low-noise amplifiers (LNAs), which simultaneously observe the sky [1]-[6]. Knowledge of the noise parameters of the LNAs as well as the part-to-part variation is important to achieve certain scientific objectives which require extremely accurate knowledge of instrumental effects [7]-[10]. The community increasingly recognizes the potential need to measure a large number of LNAs prior to deployment to accurately compute the array noise temperature of the telescope [11], [12]. The ability to measure noise parameters rapidly and accurately is of great interest in this field.

The extraction of noise parameters entails at least four measurements from which the four noise parameters may be inferred. This is usually expressed as a matrix equation

$$
A \mathbf{x}=\mathbf{t}
$$

where $A$ is an $m \times 4$ real matrix $(m \geq 4)$ containing information regarding reflection coefficients [13], [14] or the source admittances [15]-[19] presented to the device under test
(DUT); $\mathrm{x}$ is a vector containing the four noise parameters and t is the measurement vector. We refer to $A$ as the "source" matrix henceforth. Equation (1) is a standard linear algebra problem, which is solvable with the generalized inverse (also called pseudoinverse denoted by superscript.$^{+}$) [20] of $A$.

$$
\mathbf{x}^{+}=A^{+} \mathbf{t}
$$

where $\mathrm{x}^{+}$is the pseudoinverse solution and $A^{+}$is the matrix pseudoinverse of $A$. It is widely accepted that $A$ must have linearly independent columns such that $\operatorname{rank}(A)=4$; anything less is considered degenerate and requires correction. The pseudoinverse solution works for $m \geq 4$; for $m=4$, $A^{+}=A^{-1}$; whereas for $m>4$ the pseudoinverse is the least-squares solution $A^{+}=\left(A^{T} A\right)^{-1} A^{T}$.

It has been long recognized that judicious selection of "wellspread" source points (source reflection coefficient, $\Gamma_{s}$, or source admittance, $Y_{s}$ ) in $A$ permits noise parameter extraction with few (or fewer) points, which saves measurement time [21], [22]. This has been further demonstrated in the matrix inversion case $(m=4)$ [16], [17] where the resulting uncertainties are found to be comparable to $m>4$ points. A typical approach to demonstrate "well-spread" points is to suggest a constellation of points, extract the noise parameter of an example DUT and perturb the result numerically (e.g. Monte Carlo analysis) or experimentally to estimate the uncertainty (e.g., [16], [18], [21] and many others). A conclusion regarding the merit of the constellation is reached based on the resulting uncertainty estimates. This approach is widely accepted but is time-consuming and computationally expensive; it is also DUT dependent. It is noteworthy that such studies have concluded that a well-designed source matrix (with well-spread points) is able to extract noise parameters with few points and acceptable uncertainty independent of the noise parameters of the DUT [16], [17], [21]. This suggests that there is something in the source matrix itself that is quantifiable as a figure of merit.

The question of figure of merit for the source matrix based on source admittances (for brevity, $A_{Y}$ ) has been previously explored. In [18] the condition number of $A_{Y}$ was considered as figure of merit but discarded because it did not predict the resulting uncertainties. This is an unfortunate outcome because the condition number is a fundamental measure of matrix conditioning in linear algebra, which applies to $m \geq 4$. For the $m=4$ case, the determinant of $A_{Y}$ was found to be correlated to well-spread points and low uncertainties in the 
result [16]. However, it involves a normalization factor that is determined through numerical experimentation and is not clearly motivated physically. Furthermore, the determinant is not applicable to the $m>4$ case.

In this paper, we review the question of the spread of points and a quantitative measure thereof. We find that the makeup and scaling of the entries of $A$ matters greatly. We will demonstrate that the removal of the $1 /\left(1-|\Gamma|^{2}\right)$ singularity and the choice of noise-wave based parameters dramatically improve the conditioning of the matrix and put an upper bound on the $\mid$ det $\mid$, significantly clarifying the quantification of wellspread points. We discuss these in Sections II and III. Sec. IV develops the equations required to obtain the noise measurement vector compatible with the noise-wave based equation with singularity removed. In Sec. V we apply the method to noise extraction of a DUT in the SKA-Low frequency band of 50 to $350 \mathrm{MHz}$ using 7 positions in a source tuner rated for $100 \mathrm{MHz}$ to $1 \mathrm{GHz}$. The effectiveness of the selected noise matrix becomes particularly evident when evaluated over source impedance uncertainties and in the prediction of success of noise extraction at frequencies below the lowest noise tuner rating of $100 \mathrm{MHz}$.

A short 2-page summary of this work containing information in Sec. III-A has been presented in [23]. This full paper is a significant expansion of that work.

\section{BACKGROUND THEORY}

\section{A. Noise Temperature and Noise Waves}

This section discusses the physical motivation for the noise equation and its connection to similar ideas in the literature. As a starting point we express the noise temperature based on a widely accepted noise wave representation for a 2-port network [24]. The interaction of the noise temperature, noise waves and source reflection coefficient is given by

$$
k T_{n}=\frac{\boldsymbol{\alpha} C_{s} \boldsymbol{\alpha}^{H}}{1-\left|\Gamma_{s}\right|^{2}}
$$

where ${ }^{H}$ symbolizes the conjugate transpose operation, $T_{n}$ is the input-referred noise temperature in $\mathrm{K}, k$ is Boltzmann constant, $\alpha$ is a row vector and $C_{s}$ is the noise wave correlation matrix

$$
\begin{aligned}
\boldsymbol{\alpha} & =\left[\Gamma_{s}, \frac{1-\Gamma_{s} S_{11}}{S_{21}}\right] \\
C_{s} & =\left\langle\mathbf{c c}^{H}\right\rangle
\end{aligned}
$$

where $\mathbf{c}=\left[c_{1}, c_{2}\right]^{T} ; c_{1}, c_{2}$ are the noise waves emanating from the input and output of the DUT, respectively; $\langle$.$\rangle indicates the$ mean of the quantity in the brackets.

The goal of noise parameter measurement is to extract the 4 unknowns in $C_{s}:\left\langle\left|c_{1}\right|^{2}\right\rangle,\left\langle\left|c_{2}\right|^{2}\right\rangle$, the real and imaginary parts of $\left\langle c_{1} c_{2}^{*}\right\rangle$, or an equivalent combination/derivation therefrom. These parameters are inferred from at least four measurements based on (3). The numerator of (3) is of primary interest as it describes the behavior of noise sources internal to the DUT which interact with $\Gamma_{s}$.
The most common practice in noise parameter measurement is to express the left-hand side as shown in (3), that is as inputreferred quantities $T_{n}$ or noise factor $F=1+T_{n} / T_{0}\left(T_{0}=\right.$ $290 \mathrm{~K})$ as a function of $\Gamma_{s}$ [14]-[19]. However this choice is not fundamental as $T_{n}$ or $F$ are not directly measurable quantities; they are derived from other measurements, most notably the noise power recorded by the noise receiver. We can alternatively express

$$
k T_{n}^{\prime}=\boldsymbol{\alpha} C_{s} \boldsymbol{\alpha}^{H}=\left\langle|\boldsymbol{\alpha} \mathbf{c}|^{2}\right\rangle
$$

where $T_{n}^{\prime}=T_{n}\left(1-\left|\Gamma_{s}\right|^{2}\right)$ is the incident noise power at the input. $T_{n}^{\prime}$ is always a finite quantity, which removes the singularity at $\left|\Gamma_{s}\right| \rightarrow 1$. This can similarly be seen in righthand side of (6); the linear combination of noise waves is always a finite quantity. This is useful for measurements at the SKA-Low frequencies in particular those using long lowloss cables, which tend to be very low loss at tens of $\mathrm{MHz}$ [25]-[27]. Also as we will demonstrate later, the removal of this singularity clarifies the connection between the choice of $\Gamma_{s}$ and the resulting robustness of the source matrix.

Next we review key links between (6) and similar expressions in the literature. We multiply out the right-hand side of (6) resulting in

$$
k T_{n}^{\prime}=\left|\Gamma_{s}\right|^{2}\left\langle\left|c_{1}\right|^{2}\right\rangle+2 \Re\left(\Gamma_{s} \chi^{*}\left\langle c_{1} c_{2}^{*}\right\rangle\right)+|\chi|^{2}\left\langle\left|c_{2}\right|^{2}\right\rangle
$$

where $\chi=\left(1-\Gamma_{s} S_{11}\right) / S_{21}$. However, this form does not appear convenient for noise parameter extraction using $\Gamma_{s}$ variation because of its interaction with $S_{11}$ and $S_{21}$ in $\chi$. Alternatively, we can use the input-based noise waves $A_{n}, B_{n}$ discussed in [13], [28]. By comparing the noise wave definitions in [24] to [13], [28], we find

$$
\begin{aligned}
& c_{1}=S_{11} A_{n}+B_{n} \\
& c_{2}=S_{21} A_{n}
\end{aligned}
$$

The incident noise wave at the input due to the DUT noise is

$$
\boldsymbol{\alpha} \mathbf{c}=A_{n}+\Gamma_{s} B_{n}
$$

The corresponding incident noise power is

$$
k T_{n}^{\prime}=\left\langle\left|A_{n}\right|^{2}\right\rangle+\left|\Gamma_{s}\right|^{2}\left\langle\left|B_{n}\right|^{2}\right\rangle+2 \Re\left(\Gamma_{s}\left\langle A_{n}^{*} B_{n}\right\rangle\right)
$$

For ease of computation with matrices we express (10) as

$$
\begin{aligned}
k T_{n}^{\prime} & =\left\langle\left|A_{n}\right|^{2}\right\rangle+\left|\Gamma_{s}\right|^{2}\left\langle\left|B_{n}\right|^{2}\right\rangle \\
& +\Re\left(\Gamma_{s}\right) 2 \Re\left\langle A_{n}^{*} B_{n}\right\rangle-\Im\left(\Gamma_{s}\right) 2 \Im\left\langle A_{n}^{*} B_{n}\right\rangle
\end{aligned}
$$

which can be written as

$$
\begin{aligned}
k T_{n}^{\prime} & =\left(1-\left|\Gamma_{s}\right|^{2}\right)\left\langle-\left|B_{n}\right|^{2}\right\rangle+\left\langle\left|B_{n}\right|^{2}\right\rangle+\left\langle\left|A_{n}\right|^{2}\right\rangle \\
& +\Re\left(\Gamma_{s}\right) 2 \Re\left\langle A_{n}^{*} B_{n}\right\rangle-\Im\left(\Gamma_{s}\right) 2 \Im\left\langle A_{n}^{*} B_{n}\right\rangle
\end{aligned}
$$

Expressing the above as vector multiplication

$$
\begin{aligned}
T_{n}^{\prime} & =\left[1-\gamma_{s}^{2}, 1, \Re\left(\Gamma_{s}\right), \Im\left(\Gamma_{s}\right)\right] \mathbf{x} \\
\mathbf{x} & =[a, b, c, d]^{T}
\end{aligned}
$$

where $\gamma_{s}=\left|\Gamma_{s}\right|, a=\left\langle-\left|B_{n}\right|^{2}\right\rangle / k, b=\left(\left\langle\left|B_{n}\right|^{2}\right\rangle+\right.$ $\left.\left\langle\left|A_{n}\right|^{2}\right\rangle\right) / k, c=2 \Re\left\langle A_{n}^{*} B_{n}\right\rangle / k, d=-2 \Im\left\langle A_{n}^{*} B_{n}\right\rangle / k$; 
$a, b, c, d$ have units of K. Equation (13) is the same form as presented in [14] except for the scaling by $1-\gamma_{s}^{2}$. The vector $\mathbf{x}$ contains the four noise parameters to be extracted from $T_{n}^{\prime}$ measurements.

\section{B. Source Matrix and Spread of Points}

We observe the following trend in the literature. The source tuner community [17]-[19] tends to use the input admittance based expression as introduced in the original paper [15]

$$
\begin{aligned}
F & =\left[1, \frac{\left|Y_{s}\right|^{2}}{G_{s}}, \frac{1}{G_{s}}, \frac{B_{s}}{G_{s}}\right] \overline{\mathbf{x}} \\
\overline{\mathbf{x}} & =[A, B, C, D]^{T}
\end{aligned}
$$

where $Y_{s}=G_{s}+j B_{s}$ is the source admittance. The source matrix based on reflection coefficient matrix (as discussed in Sec. II-A) is preferred by non-tuner users; here the noise parameters are inferred from fast variation over a frequency band using a mismatch device [14], [25], [27], [29], [30]. Both forms are considered here. The discussion will gradually place more emphasis on the $\Gamma$-based source matrix given in Sec. II-A because it is much more directly related to a transmission line (TL) based mismatch device such as a slide screw tuner or a long cable especially over a wide band. Furthermore, there is a mathematical reason for this choice which we will discuss.

Well-spread points is a means to an end. Qualitatively we want a matrix that results in acceptable uncertainties for all reasonable DUTs for a given input uncertainty. More quantitatively, a good selection of points should lead to a source matrix with highly linearly independent columns that are well-scaled. We consider the following measures.

1) Determinant: In the $4 \times 4$ case, the matrix must be invertible. It is obvious that the matrix must have non zero determinant (det $\neq 0$ ). This is useful for hand calculation but not for numerical problem as $|\operatorname{det}|$ does not generally offer guidance regarding the relative invertibility of the matrix. For example, scaling the matrix by a constant $c$ scales the determinant by $c^{4}$. Also, maximizing $|\operatorname{det}|$ of a matrix minimizes the $|\operatorname{det}|$ of the inverse as $\operatorname{det}(A) \operatorname{det}\left(A^{-1}\right)=1$ [20]. Some measure of guidance may be obtained for a particular matrix by extensive numerical experimentation through which one learns the desirable trends of the determinant and suggests a target [16]. However, the underlying reason for that behavior is not clearly evident. We will demonstrate that removal of the $\left|\Gamma_{s}\right| \rightarrow 1$ singularity places an upper limit on $|\operatorname{det}|$ (for $\Gamma_{1}=0$ ) within a factor of a few to the ideal $|\operatorname{det}|=1$, which significantly clarifies the picture. Furthermore for the source matrix based on noise waves with singularity removed, we can derive the analytical det expression that is very highly correlated to the condition number of the matrix.

2) Condition Number: Condition number is the standard measure of the invertibility and scaling of a matrix [20]. It applies generally for $m \geq 4$.

$$
c(A)=\left\|A^{+}\right\|\|A\|=\frac{\sigma_{\max }}{\sigma_{\min }}
$$

where $\|$.$\| is the matrix norm, \|A\|=\sigma_{\max }$ is the largest and $\sigma_{\min }=1 /\left\|A^{+}\right\|$is the smallest singular value of $A$.
The cost of solving a matrix equation is the loss of digits of accuracy to roundoff errors up to the order of magnitude of $c(A), \log _{10} c(A)$ [20].

The condition number also places an upper bound of error scaling. Let the noise temperature/figure and source measurement errors be $\Delta \mathbf{t}$ and $\Delta A$, respectively. These impairments produce an error in the inferred noise parameter, $\Delta \mathbf{x}$. We express this as

$$
\begin{aligned}
\tilde{A} \tilde{\mathbf{x}} & =\tilde{\mathbf{t}} \\
(A+\Delta A)(\mathbf{x}+\Delta \mathbf{x}) & =\mathbf{t}+\Delta \mathbf{t}
\end{aligned}
$$

where . indicates the presence of error. Simplifying (18) leads to the error equation.

$$
A \Delta \mathbf{x}=\Delta \mathbf{t}-\Delta A(\mathbf{x}+\Delta \mathbf{x})
$$

with solution

$$
\Delta \mathbf{x}^{+}=A^{+}[\Delta \mathbf{t}-\Delta A \tilde{\mathbf{x}}]
$$

Using matrix norm definition and triangle inequality [20], we can show that the relative error bound is

$$
\begin{aligned}
\frac{\left\|\Delta \mathbf{x}^{+}\right\|}{\|\tilde{\mathbf{x}}\|} & \leq\left\|A^{+}\right\|\|A\|\left(\frac{\|\Delta \mathbf{t}\|}{\|A\|\|\tilde{\mathbf{x}}\|}+\frac{\|\Delta A\|}{\|A\|}\right) \\
& \leq c(A)\left(\frac{\|\Delta \mathbf{t}\|}{\|A \tilde{\mathbf{x}}\|}+\frac{\|\Delta A\|}{\|A\|}\right)
\end{aligned}
$$

where $\|\Delta \mathbf{t}\| /\|A \tilde{\mathbf{x}}\|$ is the relative input error due to noise temperature measurement and $\|\Delta A\| /\|A\|$ is the relative input error due to source measurement. The sum of the two is the total input error. Equation (21) states that $c(A)$ controls the worst case error scaling. Note that (21) by nature is a pessimistic measure; it does not predict the expected value or the variance of the error. This is the most that can be said about the resulting error based on the source matrix alone (independent of the DUT and the input errors). However, aiming for $c(A)$ as close as possible to 1 is a good strategy because it tends to equalize the singular values of $A$ making the range of possible error scaling less extreme. For example in the $m=4$ case, (21) reaches equality at $c(A)=1$ indicating no error magnification.

In [18] the condition number for $A_{Y}$ has been studied but deemed unreliable. However, we suggest that this due to the high condition number (in the few thousands as we will demonstrate) of $A_{Y}$. This means that the error bound scaling is not well constrained and not useful, e.g. relative input error of $1 \%$ corresponds to worst case output error of 1000 's \%. In contrast, we will show that the source matrix based on (12) is very well-conditioned and can reach $c(A)$ well below 10 down to a factor of a few.

For a four-column matrix it is equally straightforward to numerically compute $c(A)$ and det. However, we here strive for a more than a numerical experiment. We seek an expression resembling a design equation that measures the extent of the "well-spread" points. In this regard, the analytical expression for det is much more tractable than that of $c(A)$. Provided the det expression results in well bounded values that are highly correlated with $c(A)$, we can use that as a measure of the quality of the $4 \times 4$ matrix $A$. We explore this with the following examples. 


\section{Determinant and Condition Number of Source MATRICES}

\section{A. Noise Wave-Based Source Matrices}

Consider the noise-wave based source matrix with singularity [14]

$$
A_{\Gamma}=\left[\begin{array}{cccc}
1 & \frac{1}{1-\gamma_{1}^{2}} & \frac{\gamma_{1} \cos \theta_{1}}{1-\gamma_{1}^{2}} & \frac{\gamma_{1} \sin \theta_{1}}{1-\gamma_{1}^{2}} \\
\vdots & \vdots & \vdots & \vdots \\
1 & \frac{1}{1-\gamma_{4}^{2}} & \frac{\gamma_{4} \cos \theta_{4}}{1-\gamma_{4}^{2}} & \frac{\gamma_{4} \sin \theta_{4}}{1-\gamma_{4}^{2}}
\end{array}\right]
$$

where $\Gamma_{i}=\left|\gamma_{i}\right| \angle \theta_{i}$. As discussed in Sec. II-A, we remove the singularities by multiplying each row with $1-\gamma_{i}^{2}$

$$
A_{\Gamma m o d}=\left[\begin{array}{cccc}
1-\gamma_{1}^{2} & 1 & \gamma_{1} \cos \theta_{1} & \gamma_{1} \sin \theta_{1} \\
\vdots & \vdots & \vdots & \vdots \\
1-\gamma_{4}^{2} & 1 & \gamma_{4} \cos \theta_{4} & \gamma_{4} \sin \theta_{4}
\end{array}\right]
$$

Note in (23) a key property that every entry of the matrix is bounded as $\left|A_{\Gamma \bmod }(i, j)\right| \leq 1$.

We now consider a special case. It is expedient and wellaccepted [13], [14], [16], [17], [29], [31] that one of the source points be the reference impedance. Let $\gamma_{1}=0$ such that the first row of the source matrix is $[1,1,0,0]$. We compute and report $|\operatorname{det}|$ and $c$ of $\left.A_{\Gamma}\right|_{\gamma_{1}=0}$ in Fig. 1 and $\left.A_{\Gamma_{m o d}}\right|_{\gamma_{1}=0}$ in Fig. 2 using $10^{5}$ random trials with uniform probability over the Smith chart. The only difference between Fig. 1 and Fig. 2 is the removal of $1 /\left(1-\left|\Gamma_{s}\right|^{2}\right)$ singularity in the latter. We now closely examine the correlation between det and $c$. Fig. 1 does not show strong correlation nor convergence between $c$ and $|\operatorname{det}|$. In contrast, Fig. 2 shows that $\mid$ det $\mid$ has a well-defined maximum and $|\operatorname{det}|$ is very strongly anti-correlated with its condition number, $c$. High $|\operatorname{det}|$ strongly predicts low $c$, which is the desired behavior. The maximum $\mid$ det $\mid$ converges with the lowest $c$ at about $\sim 2.5$ for both, as seen near the bottom right corner of the plot. The removal of the singularity results in the convergence between $c$ and $|\operatorname{det}|$ for this important special case.

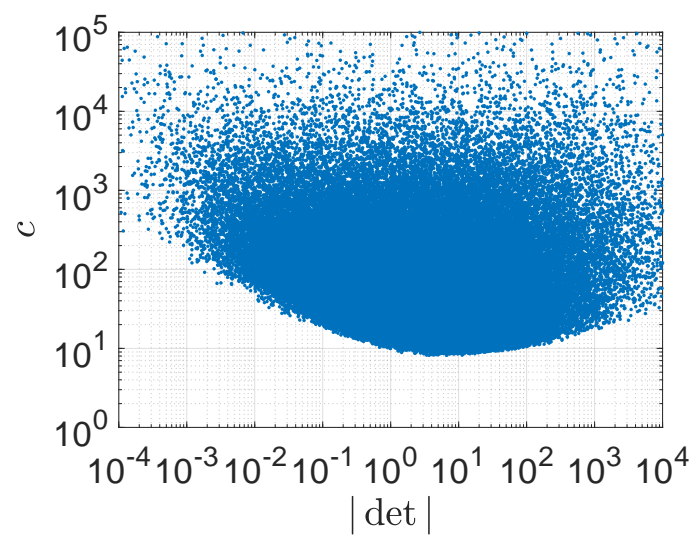

Figure 1. Plot of $|\operatorname{det}|$ vs. the condition number for the unmodified $\left.A_{\Gamma}\right|_{\gamma_{1}=0}$ with $10^{5}$ random trials. The $|\operatorname{det}|$ is computed numerically as it is not known in analytical form. There is no convergence between $c$ and det.

We now examine the physical reason for the low $c$ and high $|\operatorname{det}|$. Because $c$ and $|\operatorname{det}|$ converge in the desirable

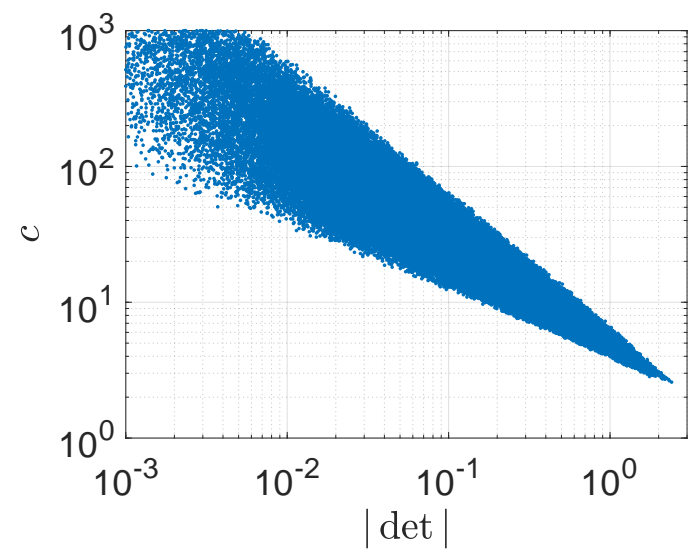

Figure 2. Plot of $|\operatorname{det}|$ vs. the condition number for $A_{\Gamma_{m o d}} \mid \gamma_{1}=0$ with $10^{5}$ random trials. The lowest condition number converges to the highest $|\operatorname{det}|$. Therefore, high $|\operatorname{det}|$ is indicative of a well conditioned matrix.

region and $|\operatorname{det}|$ is much more readily computed analytically, we derive $\operatorname{det}\left(\left.A_{\Gamma_{m o d}}\right|_{\gamma_{1}=0}\right)$ by Gaussian elimination and multiplication of the pivots [20]. Following this process and algebraic simplification, it can be shown that

$$
\mid \text { det }\left|=\gamma_{2} \gamma_{3} \gamma_{4}\right| \gamma_{3} \sin \theta_{42}-\gamma_{4} \sin \theta_{32}-\gamma_{2} \sin \theta_{43} \mid
$$

where $|\operatorname{det}|=\operatorname{det}\left(\left.A_{\Gamma_{\text {mod }}}\right|_{\gamma_{1}=0}\right)$ for brevity; $\theta_{i j}$ are relative angles, e.g. $\theta_{43}=\theta_{4}-\theta_{3}$ and similarly with $\theta_{42}$ and $\theta_{32}$. Equation (24) suggests:

1) Only the relative phases matter. The $|\operatorname{det}|$ is rotationally-invariant with respect to the center of the Smith chart.

2) The maximum $\mid$ det $\mid$ of 2.598 occurs for $\gamma_{2}=\gamma_{3}=$ $\gamma_{4}=1$ and $\theta_{32}= \pm 120^{\circ}$ and $\theta_{42}= \pm 240^{\circ}$. It is interesting to note that the source point distribution in this way, i.e. phase difference between points of $120^{\circ}$ for $\gamma_{2,3,4}=1$ and $\gamma_{1}=0$ is in fact the maximum possible spread of points with a passive source. Equation (24) provides a solid basis for the conjecture based on numerical experiments that the points must be "wellspread" over the Smith chart [16], [18], [21].

We have verified (24) using the symbolic math package in MATLAB and by comparing the result with numerical computation.

Table I

EXAMPLES OF $|\operatorname{det}|$ FOR $A_{\Gamma_{\text {mod }}} \mid \gamma_{1}=0$ WHERE $\gamma=\gamma_{1}=\gamma_{2}=\gamma_{3}$ AND $\theta=\theta_{32}=\theta_{43}$.

\begin{tabular}{|l|ccccc|}
\hline$\gamma$ & $30^{\circ}$ & $60^{\circ}$ & $90^{\circ}$ & $120^{\circ}$ & $150^{\circ}$ \\
\hline 0.4 & 0.003 & 0.022 & 0.051 & 0.067 & 0.048 \\
0.6 & 0.017 & 0.112 & $\mathbf{0 . 2 5 9}$ & $\mathbf{0 . 3 3 7}$ & 0.242 \\
0.8 & 0.055 & $\mathbf{0 . 3 5 5}$ & $\mathbf{0 . 8 1 9}$ & $\mathbf{1 . 0 6 4}$ & $\mathbf{0 . 7 6 4}$ \\
1 & 0.134 & $\mathbf{0 . 8 6 6}$ & $\mathbf{2 . 0 0 0}$ & $\mathbf{2 . 5 9 8}$ & $\mathbf{1 . 8 6 6}$ \\
\hline
\end{tabular}

To study a few representative cases, Tab. I reports the computed $|\operatorname{det}|$ for $\left.A_{\Gamma_{m o d}}\right|_{\gamma_{1}=0}$ for equal $\gamma_{2,3,4}$ and equal phase steps. The boldface values indicate desirable $\gamma$ and $\theta$ entries for which $\mid$ det $\mid$ are within one order of magnitude from the maximum. For the same $\gamma$ and $\theta$, the computed conditions 
numbers corresponding to the boldface entries are similarly favorable at approximately 10 or less as shown in the next Tab. II.

Table II

EXAMPLES OF $c$ FOR $\left.A_{\Gamma_{m o d}}\right|_{\gamma_{1}}=0 \gamma=\gamma_{1}=\gamma_{2}=\gamma_{3}$ AND
$\theta=\theta_{32}=\theta_{43}$.

\begin{tabular}{|l|ccccc|}
\hline$\gamma$ & $30^{\circ}$ & $60^{\circ}$ & $90^{\circ}$ & $120^{\circ}$ & $150^{\circ}$ \\
\hline 0.4 & 211.04 & 48.77 & 27.62 & 25.64 & 25.96 \\
0.6 & 94.57 & 21.29 & 10.92 & 9.89 & 10.06 \\
0.8 & 54.43 & 11.94 & 5.41 & 4.65 & 4.77 \\
1 & 37.19 & 7.94 & 3.23 & 2.48 & 3.01 \\
\hline
\end{tabular}

\section{B. Singularity Removal from Admittance-Based Source Matrix}

For comparison, we have also considered the admittance based source matrix as per (15) as well the singularity removal thereof. In this context,

$$
1-\gamma_{s}^{2}=\frac{4 Y_{o} G_{s}}{\left(G_{s}+G_{o}\right)^{2}+B_{s}^{2}}
$$

where $Y_{o}=1 / Z_{o}$ is the real reference admittance. The equation with singularity removed becomes

$$
F^{\prime}=\left(1-\gamma_{s}^{2}\right)\left[1, \frac{\left|Y_{s}\right|^{2}}{G_{s}}, \frac{1}{G_{s}}, \frac{B_{s}}{G_{s}}\right] \overline{\mathbf{x}}
$$

where $F^{\prime}=F\left(1-\gamma_{s}^{2}\right)$. The measurement matrix is formed by stacking $m$ rows of (26) for different $Y_{s}=G_{s}+j B_{s}$ values.

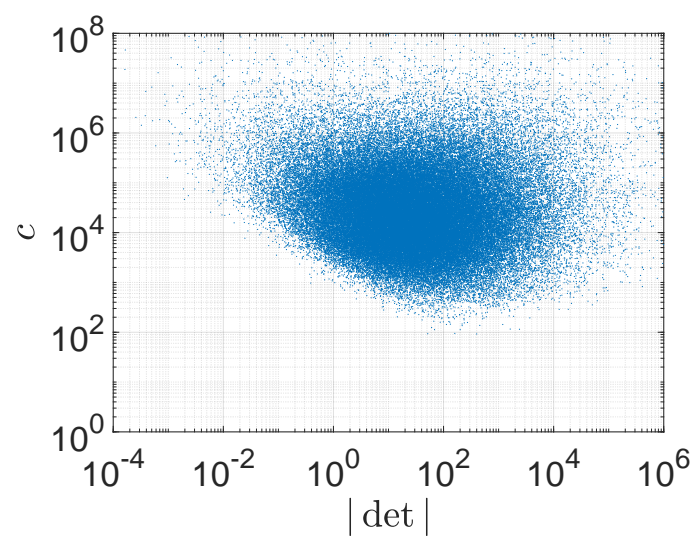

Figure 3. Plot of $\mid$ det $\mid$ vs. the condition number for the unmodified source matrix based on admittances $A_{Y}$ with $\gamma_{1}=0$ and $10^{5}$ random trials. Again, there is no convergence between $c$ and det.

We repeat the same computation with $10^{5}$ random trials with the corresponding source matrices based on admittances for $\gamma_{1}=0\left(Y_{s}=Y_{o}\right)$ with and without singularity removal $\left(A_{Y_{\text {mod }}}\right.$ and $\left.A_{Y}\right)$. Fig. 3 shows $|\operatorname{det}|$ vs. $c$ for the unmodified matrix where no clear trend is detected. Singularity removal results in an upper bound of $|\operatorname{det}| \approx 40$ as shown in Fig. 4. There is some anti-correlation between $|\operatorname{det}|$ vs. $c$ however the maximum $|\operatorname{det}|$ does not coincide with the minimum $c$. Furthermore, the low $c$ values are in $\sim 1000$ 's making the error scaling upper bound far less constrained than the previous section.

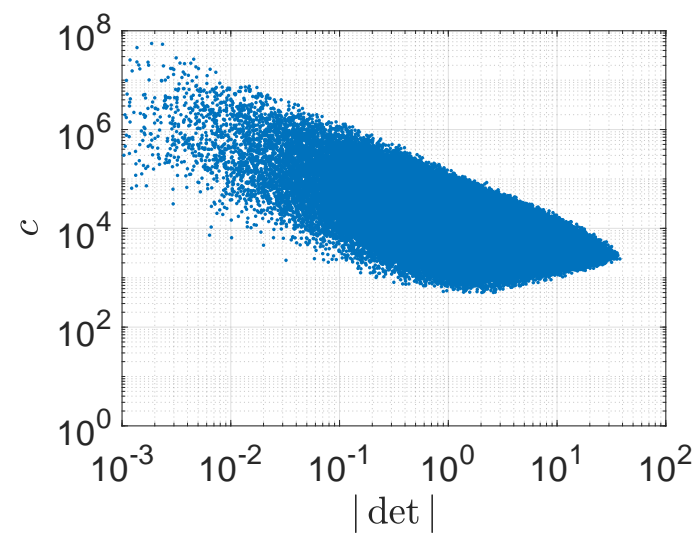

Figure 4. Plot of $|\operatorname{det}|$ vs. the condition number source matrix based on admittances $A_{Y_{m o d}}$ with $\gamma_{1}=0$ and singularity removed with $10^{5}$ random

\begin{tabular}{|c|c|c|c|c|c|}
\hline EXAMPLES OI & $\operatorname{det} \mid$ & $\begin{array}{l}\mathrm{R} A_{Y_{n}} \\
\theta=\end{array}$ & $\begin{array}{l}\text { e III } \\
\text { WHEF } \\
=\theta_{4}\end{array}$ & $\gamma=\gamma$ & $=\gamma_{2}$ \\
\hline & $30^{\circ}$ & $60^{\circ}$ & $90^{\circ}$ & $120^{\circ}$ & $150^{\circ}$ \\
\hline 0.4 & 0.05 & 0.35 & 0.82 & 1.06 & 0.76 \\
\hline 0.6 & 0.28 & 1.80 & 4.15 & 5.39 & 3.87 \\
\hline 0.8 & 0.88 & 5.68 & 13.11 & 17.03 & 12.23 \\
\hline 1 & 2.14 & 13.86 & 32.00 & 41.57 & 29.86 \\
\hline
\end{tabular}
trial.

We see the $|\operatorname{det}|$ values in Tab. III within an order of magnitude of the maximum occupy the same entries as Tab. I. However, the $c$ values in Tab. IV show a decreasing trend towards the bottom left of the table for which the distributions of points are less spread out. We suggest this is the reason that $|\operatorname{det}|$ is found to be a good figure of merit [16] whereas $c$ is found not useful [18] in previous studies. We also suggest that the determinant normalization in [16] is in fact similar in effect to scaling with $1-\gamma^{2}$ in (25).

\section{Measurement Equations}

We now demonstrate how to obtain $T_{n}^{\prime}$ in a real measurement. The primary focus is on the cold source method, which we will use to extract the noise parameters of an active DUT in the next section. We will also illustrate the slight modifications required to obtain the $T_{n}^{\prime}$ from the Y-factor (hot/cold) method.

\section{A. Cold Source}

Our discussion is based on the setup depicted in Fig. 5. The displayed noise power in the RX due to the DUT cascaded

Table IV
EXAMPLES OF $c$ FOR $\left.A_{Y_{m o d}}\right|_{\gamma_{1}=0}$ WHERE $\gamma=\gamma_{1}=\gamma_{2}=\gamma_{3}$ AND
$\theta=\theta_{32}=\theta_{43}$.

\begin{tabular}{|l|ccccc|}
\hline$\gamma$ & $30^{\circ}$ & $60^{\circ}$ & $90^{\circ}$ & $120^{\circ}$ & $150^{\circ}$ \\
\hline 0.4 & 28,612 & 12,400 & 11,145 & 10,250 & 9,398 \\
0.6 & 8,435 & 5,341 & 5,572 & 5,086 & 4,610 \\
0.8 & $2,871 \leftarrow$ & $2,982 \leftarrow$ & 3,589 & 3,312 & 3,005 \\
1 & $917 \leftarrow$ & $2,077 \leftarrow$ & 2,865 & 2,725 & 2,496 \\
\hline
\end{tabular}




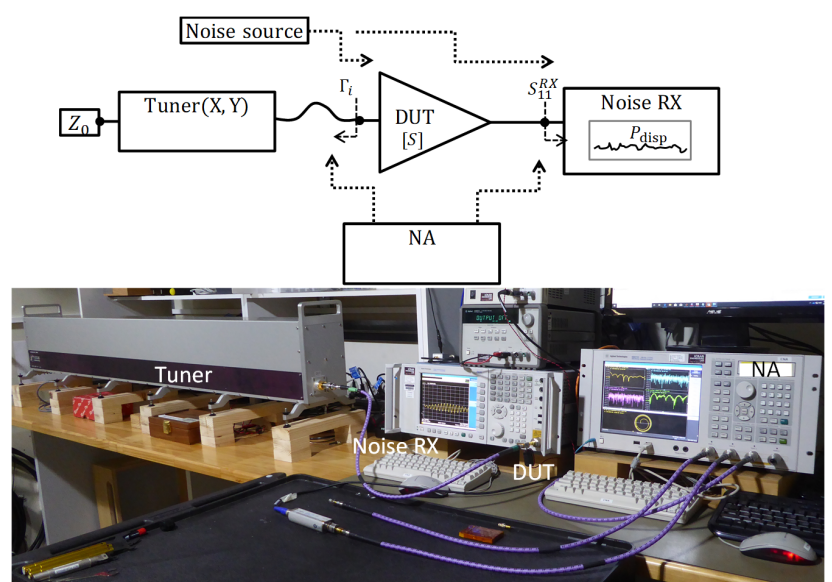

Figure 5. Example measurement setup. The tuner presents the $\Gamma_{i}$ to the input of the DUT. The noise receiver (RX) measures the noise produced at the output of the DUT. The NA is network analyzer with which we measure the tuner $\Gamma_{i}$, DUT $S$ parameters and the RX input reflection coefficient. $P_{d i s p}$ is the power measured by the noise receiver. The tuner is connected to the input of the DUT using a $1 \mathrm{~m}$ low-loss Gore MFR 541Q2 cable. The RX is a Keysight PXA N9030A and the NA is Keysight ENA E5071C calibrated with an economy cal kit $85052 \mathrm{D}$. The noise source is Keysight 346A with $\sim 5.5 \mathrm{~dB}$ ENR for calibrating the noise RX and the DUT connected to the noise RX.

with the RX $\left(T_{t o t}\right)$ and the ambient temperature source $\left(T_{a m b}\right)$ with reflection coefficient $\Gamma_{i}$ is

$$
P_{\text {disp:tot }}=\left[T_{a m b}+T_{\text {tot: } \Gamma_{i}}\right] G_{t}^{\Gamma_{i}} G_{p}^{R X} k B
$$

where $G_{p}^{R X} B$ is the gain-bandwidth product of the noise receiver (obtained from noise receiver calibration using the noise source [27]) and $G_{t}^{\Gamma_{i}}$ is the transducer gain of the DUT

$$
\begin{aligned}
G_{t}^{\Gamma_{i}} & =\frac{1-\left|\Gamma_{i}\right|^{2}}{\left|1-\Gamma_{i} \Gamma_{\mathrm{IN}}\right|^{2}}\left|S_{21}\right|^{2} \frac{1-\left|S_{11}^{R X}\right|^{2}}{\left|1-S_{11}^{R X} S_{22}\right|^{2}} \\
& =M_{i n 0}^{\Gamma_{i}} G_{t 0}
\end{aligned}
$$

where $M_{i n 0}^{\Gamma_{i}}=\left(1-\left|\Gamma_{i}\right|^{2}\right) /\left|1-\Gamma_{i} \Gamma_{\mathrm{IN}}\right|^{2}$ is a mismatch factor dependent on $\Gamma_{i}$ and $G_{t 0}$ is a DUT gain factor independent of $\Gamma_{i} ; \Gamma_{\mathrm{IN}}$ is the input reflection coefficient of the DUT connected to the RX. $G_{t 0} G_{p}^{R X} B$ is measured using the noise source connected to the input of the DUT

$$
G_{t 0} G_{p}^{R X} B=\frac{\left(P_{\text {disp:tot }}^{O N}-P_{\text {disp:tot }}^{O F F}\right) / k}{T_{O N} M_{i n 0}^{O N}-T_{O F F} M_{i n 0}^{O F F}}
$$

where $P_{\text {disp:tot }}^{O N / O F F}$ is the displayed power due to the noise source in the ON/OFF state, $T_{O N}$ is the noise temperature of noise source in the ON state, $T_{O F F}=T_{a m b}$, and

$$
M_{i n 0}^{O N / O F F}=\frac{\left(1-\left|\Gamma_{O N / O F F}\right|^{2}\right)\left(1-\left|S_{11}^{\mathrm{RX}}\right|^{2}\right)}{\left|1-S_{11}^{\mathrm{RX}} \Gamma_{O N / O F F}\right|^{2}}
$$

is the mismatch factor due to the noise source in the ON/OFF condition measured using the network analyzer (NA). This results in

$$
T_{t o t: \Gamma_{i}}^{\prime}=\frac{\left|1-\Gamma_{i} \Gamma_{\mathrm{IN}}\right|^{2} P_{d i s p: t_{t o t}}}{G_{t 0} G_{p}^{R X} B k}-T_{a m b}^{\prime}
$$

where ' indicates multiplication with $1-\gamma_{i}^{2}$. For certain DUTs and RXs for which $\Gamma_{\text {IN }}$ measurement using an NA is not practicable due to RX saturation, an alternative form may be used $\left|1-\Gamma_{i} \Gamma_{\mathrm{IN}}\right|^{2}=\left|1-\Gamma_{i} S_{11}\right|^{2}\left|1-S_{11}^{R X} \Gamma_{\text {out }_{i}}\right|^{2} /\left|1-S_{11}^{R X} S_{22}\right|^{2}$ where $\Gamma_{\text {out }_{i}}$ is the output reflection coefficient of the DUT connected to $\Gamma_{i}$. Finally, the quantity of interest is

$$
T_{D U T: \Gamma_{i}}^{\prime}=T_{t o t: \Gamma_{i}}^{\prime}-\frac{T_{R X: \Gamma_{o u t_{i}}}^{\prime}}{G_{a_{i}}}
$$

where

$$
G_{a_{i}}=\frac{1-\left|\Gamma_{i}\right|^{2}}{\left|1-\Gamma_{i} S_{11}\right|^{2}} \frac{\left|S_{21}\right|^{2}}{1-\left|\Gamma_{\text {out }_{i}}\right|^{2}}
$$

is the DUT available gain and $T_{R X: \Gamma_{\text {out }}}^{\prime}$ is the noise temperature (DUT input-terminal referred) of the RX seeing $\Gamma_{\text {out }_{i}}$. $T_{R X: \Gamma_{\text {out }_{i}}}^{\prime}$ is calculated based on noise parameters of the RX extracted during RX calibration [27] using the same tuner positions; this process is essentially the same as the foregoing discussion but with the DUT removed. Note that $\gamma_{i} \rightarrow 1$ in $T_{R X: \Gamma_{\text {out }}}^{\prime}$ and in (33) cancel each other out. For a DUT with high $G_{a_{i}}$, the correction factor $T_{R X: \Gamma_{\text {out }_{i}}}^{\prime} / G_{a_{i}}$ is small compared to $T_{t o t: \Gamma_{i}}^{\prime}$ in (32).

The equations in this subsection are readily applicable to the open cable measurement [27] if desired.

\section{B. Modification for the Y-Factor Method}

This subsection briefly illustrates that the $T_{n}^{\prime}$ quantity is measurable with the Y-factor method. For this measurement, the noise source is connected to tuner input in Fig. 5. The displayed power can be written as

$$
P_{\text {disp:tot }}^{O N / O F}=\left[\hat{T}_{O N / O F F}^{\prime}+T_{t o t: \Gamma_{i}}^{\prime}\right] \hat{G}_{t o t: \Gamma_{i}} k B
$$

where $\hat{T}_{O N / O F F}^{\prime}=\hat{T}_{O N / O F F}\left(1-\gamma_{i: O N / O F F}^{2}\right)$ is the noise temperature of the incident power at the DUT input (tuner output) for the noise source in the ON/OFF state; note that $\hat{T}_{O N / O F F}$ is influenced by the tuner loss and is not equal to the noise source temperature $T_{O N / O F F}$. The total gain is

$$
\hat{G}_{t o t: \Gamma_{i}}=\frac{G_{t 0} G_{p}^{R X}}{\left|1-\Gamma_{i} \Gamma_{\mathrm{IN}}\right|^{2}}
$$

The Y-factor is

$$
Y_{\text {tot }}=\frac{P_{\text {disp:tot }}^{O N}}{P_{{\text {disp: }: t_{i}}_{i}}^{O F F}} \approx \frac{\hat{T}_{O N}^{\prime}+T_{t o t: \Gamma_{i}}^{\prime}}{\hat{T}_{O F F}^{\prime}+T_{\text {tot }: \Gamma_{i}}^{\prime}}
$$

where the approximation comes from assuming identical $\Gamma_{i}$ in the ON/OFF state; correction can be applied if desired. The cascaded noise is

$$
T_{t o t: \Gamma_{i}}^{\prime}=\frac{\hat{T}_{O N}^{\prime}-Y_{t o t} \hat{T}_{O F F}^{\prime}}{Y_{t o t}-1}
$$

Receiver noise correction can be performed using (32). 


\section{Measurement Example}

We further demonstrate the method above with measurement of Mini Circuits ZX60-3018G+, a broadband amplifier with nominal noise figure of $2.61 \mathrm{~dB}$ at $100 \mathrm{MHz}$ as the DUT. We use the cold source measurement method and a Focus Microwaves CCMT-101 single-probe slide screw tuner as shown in Fig. 5. The objective is to extract the DUT noise parameters in the SKA-Low band of 50 to $350 \mathrm{MHz}$ which is partly below the CCMT-101 frequency range of $100 \mathrm{MHz}$ to $1 \mathrm{GHz}$.

\section{A. Selection of Tuner Positions}

The CCMT-101 consists of a single probe with two degrees of freedom: vertical $(Y)$ and and horizontal $(X)$. The probe can be represented as a TL with variable characteristic impedance of $2.8 \Omega \lesssim Z_{p} \leq 50 \Omega$ (adjustable by setting a value $4720 \geq$ $Y \geq 0$ ) followed with a $Z_{0}=50 \Omega \mathrm{TL}$ with adjustable length $0 \leq l_{X} \leq 1.5 \mathrm{~m}$ which is represented by a value $0 \leq X \leq$ 10060 . The tuner length of $1.5 \mathrm{~m}(0.5 \lambda$ at $100 \mathrm{MHz})$ means that it has full phase coverage over the Smith chart whereas at $50 \mathrm{MHz}$ it only has half phase coverage. As seen by the input of the DUT, $Y$ controls the magnitude of the tuner reflection coefficient, $\gamma_{i}$, while $X$ determines the phase of the reflection coefficient, $\theta_{i}$; the higher the $Y$ the higher the $\gamma_{i}$; the higher the $X$ the farther the rotation about the center of the Smith chart [32].
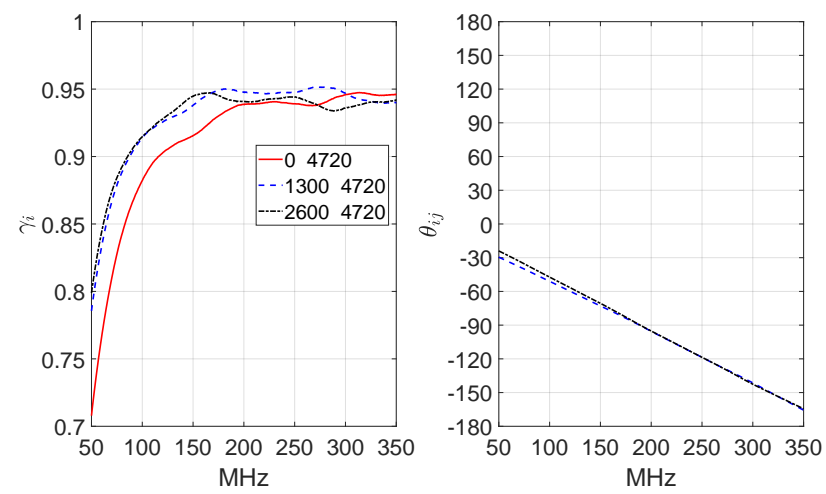

Figure 6. $\Gamma_{i}$ for $Y=4720$ and $X=0,1300,2600$. Two relative angles $\theta_{43}, \theta_{32}$ as per (24) are computed. The blue dashed line is between $X=0$ and $X=1300$; the solid black is between $X=1300$ and $X=2600$. This is the "high" band setting.

As informed by $A_{\Gamma_{m o d}}$ and (24), the highest $\mid$ det $\mid$ and the lowest $c$ occur for maximum spread of points over the Smith chart, which suggests high $\gamma$ and maximum angular separation. For the CCMT-101, we select $Y=4720$ which corresponds to the highest $\gamma$. The $X$ positions must be selected for adequate phase separation of the points over frequency as discussed next.

Taking $X=0,1300,2600$, we obtain $\Gamma_{i}$ distribution as shown in Fig. 6. With this setting (called "high" band), we obtain non zero determinant and condition number $\lesssim 100$ in Fig. 7 which suggest an invertible matrix over the entire SKALow band. However, the low determinant in Fig. 7 and high condition number in Fig. 6 at $\sim 50 \mathrm{MHz}$ indicate the need

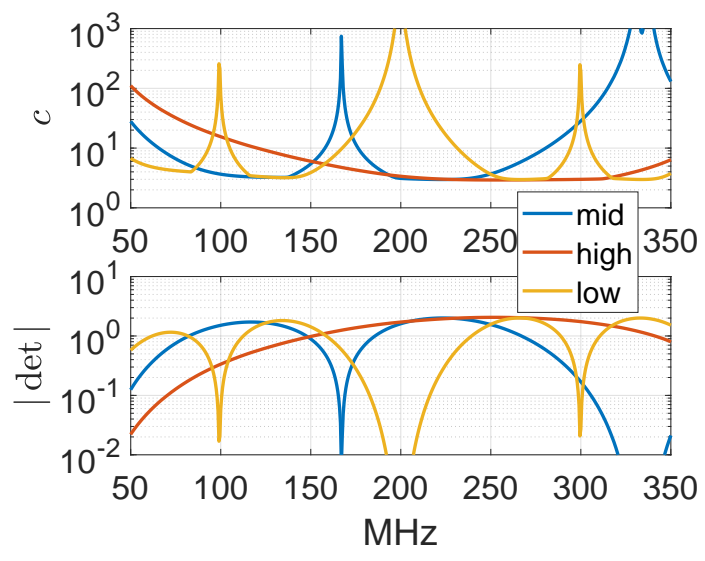

Figure 7. Determinants and conditions numbers for the 3 bands: low, mid, high.

for improvement in the phase separation between points at low frequencies. We achieve this by two additional sets of $\Gamma$ 's shown in Fig. 8 and Fig. 9. The "low" band setting with $Y=4720$ and $X=0,5000,10000$ maximizes the $X$ extent of the tuner resulting in excellent matrix conditioning at $50 \mathrm{MHz}$. However, this setting exhibits a singularity at approximately $100 \mathrm{MHz}$, which is addressed by the "mid" band setting with $Y=4720$ and $X=0,3000,6000$. The selection of $X$ positions as described achieves phase separation of approximately $90^{\circ}$ to $150^{\circ}$ for at least one of "low", "mid' or "high" tuner setting over the entire SKA-Low band.
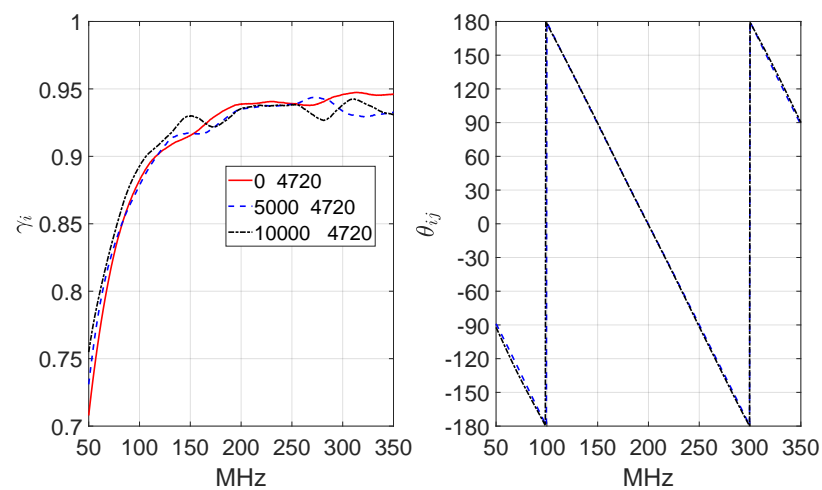

Figure 8. $\Gamma_{i}$ for $Y=4720$ and $X=0,5000,10000$. Two relative angles $\theta_{43}, \theta_{32}$ as per (24) are computed. The blue dashed line is between $X=0$ and $X=5000$; the solid black is between $X=5000$ and $X=10000$. This is the "low" band setting.

\section{B. Measurement Results and Discussions}

The measurement is taken from 10 to $400 \mathrm{MHz}$ with 781 points $(500 \mathrm{kHz}$ step) with resolution bandwidth of $1 \mathrm{MHz}$ using the equations discussed in Sec. IV-A. The noise RX takes an average over 10 thousand sweeps per measurement. The $S$-parameters are measured using the NA at matching frequency points and bandwidth. We use high power setting to measure the tuner $\Gamma_{i}$ and low power $(-35 \mathrm{dBm})$ with 256 averaging to measure the DUT and the input of the RX. The 

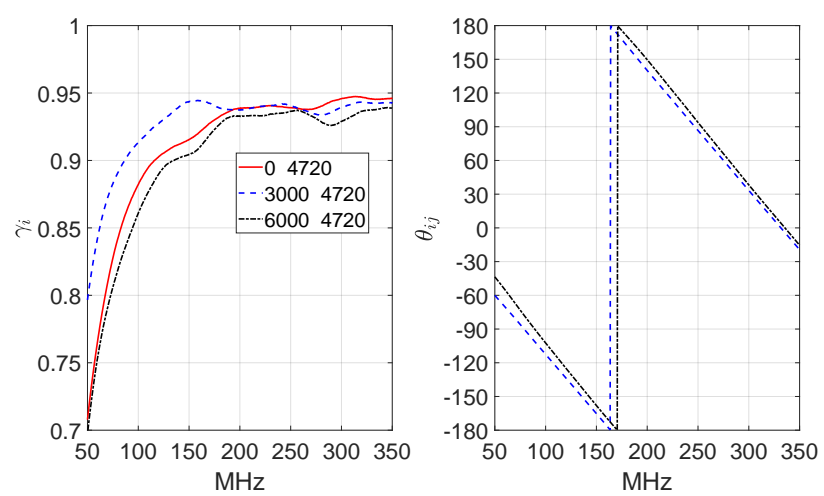

Figure 9. $\Gamma_{i}$ for $Y=4720$ and $X=0,3000,6000$. Two relative angles $\theta_{43}, \theta_{32}$ as per (24) are computed. The blue dashed line is between $X=0$ and $X=3000$; the solid black is between $X=3000$ and $X=6000$; there are two $\theta_{i j}$ areas of singularity to avoid: $\sim 0^{\circ}$ and $\sim \pm 180^{\circ}$. This is the "mid" band setting.

$T_{1}^{\prime}$ for $\Gamma_{1}=0$ is measured using a $50 \Omega$ calibration standard while the rest of the source points are taken using the CCMT101 with the 3 bands described earlier.

The solution to the matrix equation

$$
\left[\left.A_{\Gamma_{\text {mod }}}\right|_{\gamma_{1}=0}\right] \mathbf{x}=\mathbf{t}
$$

are the noise-wave based parameters $a, b, c, d$ shown in Fig. 10 as mean and standard deviation computed with $10 \mathrm{MHz}$ sliding window (20 points). The high band setting indeed returns meaningful solutions throughout the SKA-Low band. The high standard deviation below $100 \mathrm{MHz}$ is fully expected as $c$ and $|\operatorname{det}|$ in Fig. 7 suggest. The low band setting significantly reduces the standard deviation in the solution at $50-60 \mathrm{MHz}$. This is clearly evident in the relative errors in Fig. 11 where the vector norm of the solution is

$$
\|\mathbf{x}\|=\sqrt{\mathbf{x}^{T} \mathbf{x}}=\sqrt{a^{2}+b^{2}+c^{2}+d^{2}}
$$

where $\Delta\|\mathbf{x}\|,\langle\|\mathbf{x}\|\rangle$ are the standard deviation and mean, respectively, over the sliding window.

Fig. 11 also shows that low relative errors highly coincide with low $c$ and high $|\operatorname{det}|$. Consequently, low $c$ or high | det $\mid$ could be used interchangeably to select the solution with high probability of least error. The resulting noise parameters selected using lowest $c$ and max. $\mid$ det $\mid$ are shown in Fig. 12. There is negligible difference between the two.

\section{Comparison with Admittance-Based Source Matrix}

For comparison, we solve exactly the same problem using the admittance-based source matrix [16]

$$
\left[\left.A_{Y}\right|_{\gamma_{1}=0}\right] \overline{\mathbf{x}}=\mathbf{f}
$$

where $\mathbf{f}=\left[F_{1}, F_{2}, F_{3}, F_{4}\right]^{T}$ is the vector of measured noise figures and $\overline{\mathrm{x}}=[A, B, C, D]^{T}$ are the solutions; note that $[A, B, C, D]^{T} \neq[a, b, c, d]^{T}$ and

$$
\left.A_{Y}\right|_{\gamma_{1}=0}=\left[\begin{array}{cccc}
1 & Y_{o} & \frac{1}{Y_{o}} & 0 \\
\vdots & \vdots & \vdots & \vdots \\
1 & \frac{\left|Y_{4}\right|^{2}}{G_{4}} & \frac{1}{G_{4}} & \frac{B_{4}}{G_{4}}
\end{array}\right]
$$
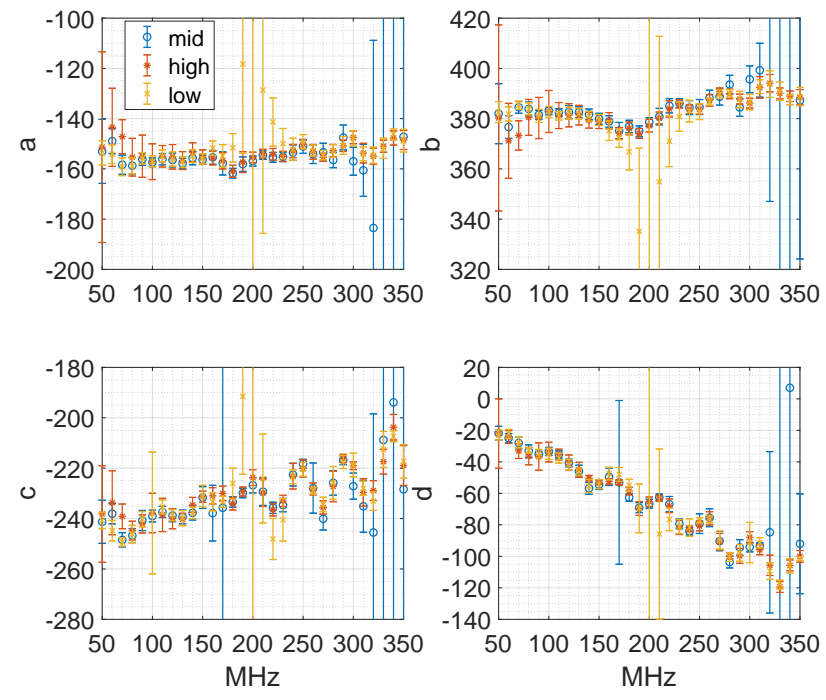

Figure 10. Noise-wave based noise parameter solutions for the low, mid and high band settings. The mean and standard deviations are computed using $10 \mathrm{MHz}$ sliding window (20 points). The unit is $\mathrm{K}$.

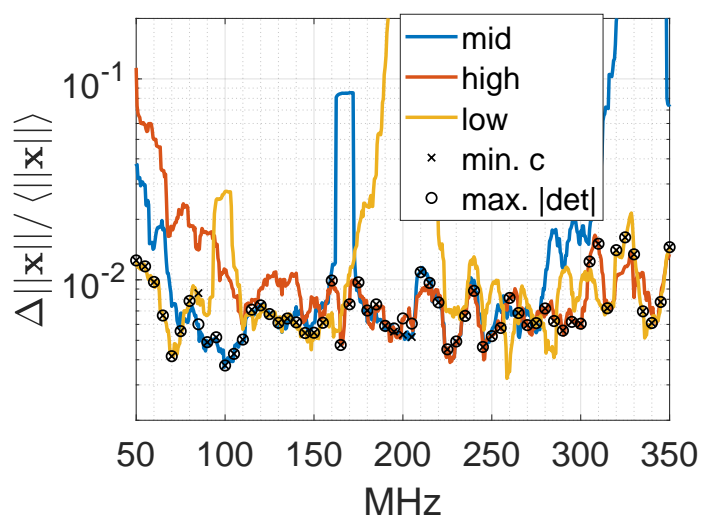

Figure 11. Relative output error computed based on standard deviation of the norm of the solution, $\|\mathbf{x}\|$, divided by the mean of the same using $10 \mathrm{MHz}$ sliding window (20 points). The black markers refer to solutions selected based on $\min c$ and max. $|\operatorname{det}|$.

The vector solution $\overline{\mathbf{x}}=[A, B, C, D]^{T}$ has been converted to noise parameters $T_{\min }, N, \Gamma_{\text {opt }}$ and then to $\mathbf{x}=[a, b, c, d]^{T}$ using conversion formulas in [14]. The relative errors are computed the same way as in Sec. V-B and reported in Fig. 13. Overall, the relative errors are very similar. However, there are divergences in the results selected by min. $c$ and max. | det |. At $220 \mathrm{MHz}$ the min. $c$ selects the solution with highest standard deviation. The results suggest that max. $|\operatorname{det}|$ is a more reliable figure of merit than min. $c$ as consistent with previous findings [16], [18] and Sec. II-B.

It is important to note that the tuner positions have been selected based on $\left.A_{\Gamma_{m o d}}\right|_{\gamma_{1}=0}$ in Sec. V-A for which both $c$ and $1 /|\operatorname{det}|$ converge. This significantly clarifies the selection process. If we only had $A_{Y}$, we would have had to select tuner positions based on Fig. 14. This would have been a much more ambiguous process involving trial-and-error. For example, recall that $c$ has been deemed unreliable in favor of $|\operatorname{det}|$. How would we know if noise parameter extraction at 

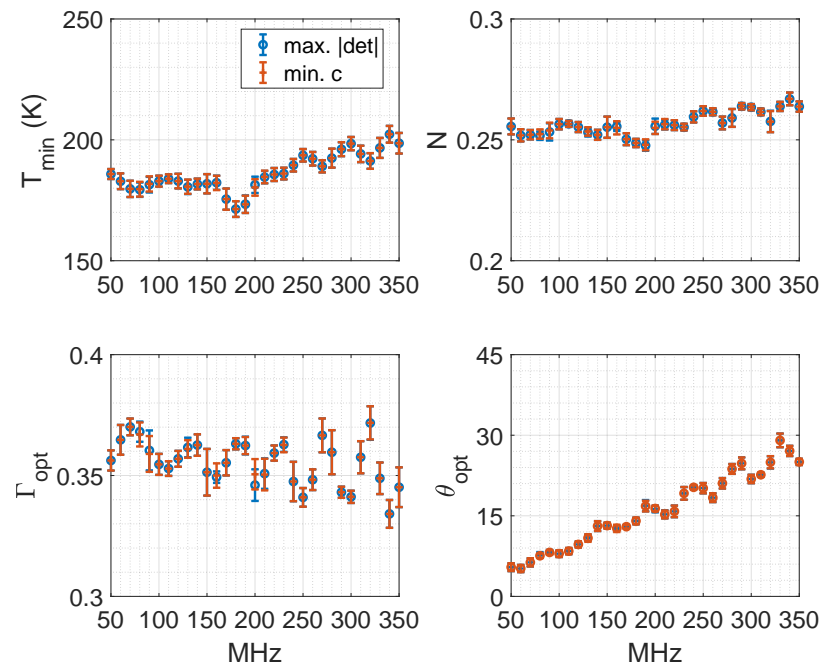

Figure 12. Noise parameters for the DUT selected using max. $|\operatorname{det}|$ and min. $c$ of $A_{\Gamma_{\text {mod }}} . N=R_{n} G_{o p t}$

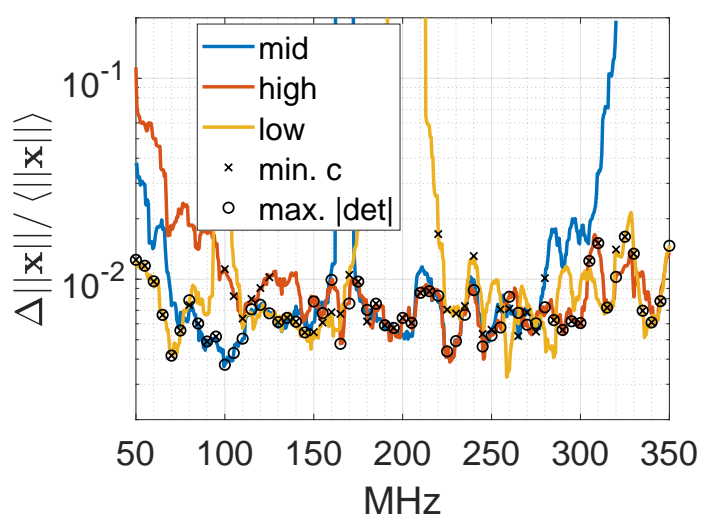

Figure 13. Relative output error using $A_{Y}$ computed based on standard deviation of the norm of the solution, $\|\mathbf{x}\|$, divided by the mean of the same using $10 \mathrm{MHz}$ sliding window (20 points). The black markers refer to solutions selected based on min $c$ and max. $|\operatorname{det}|$.

$50 \mathrm{MHz}$ is feasible? At $50 \mathrm{MHz}$ the low band setting produces $|\operatorname{det}| \approx 100$ whereas the maximum $|\operatorname{det}|$ is approximately 25000. It is unclear if this is good enough.

Contrast this with Fig. 7. At $50 \mathrm{MHz} c \approx 6$ and $|\operatorname{det}| \approx 0.6$. The minimum $c$ is approximately 3 and the maximum $|\operatorname{det}|$ is 2 . This suggests that the result should be comparable to the best tuner positions to within a factor of $\sim 2$ to 3 . Indeed at $50 \mathrm{MHz}$ the relative error standard deviation is $\approx 0.01$, which is comparable to $0.003-0.01$ relative errors selected using min. $c$ and max. $|\operatorname{det}|$ (Fig. 11).

\section{Including Source Matrix Errors}

The difference due to the selection of $\left.A_{\Gamma_{\text {mod }}}\right|_{\gamma_{1}=0}$ or $\left.A_{Y}\right|_{\gamma_{1}=0}$ matrix becomes more evident when we include the relative output error due to error in the source matrix. To obtain $\Gamma$ errors, we cycle through all the prescribed tuner positions 100 times by keeping $Y=4720$ constant and while moving $X$ back and forth. The resulting errors reported in Fig. 15 are taken as the difference between the measurements
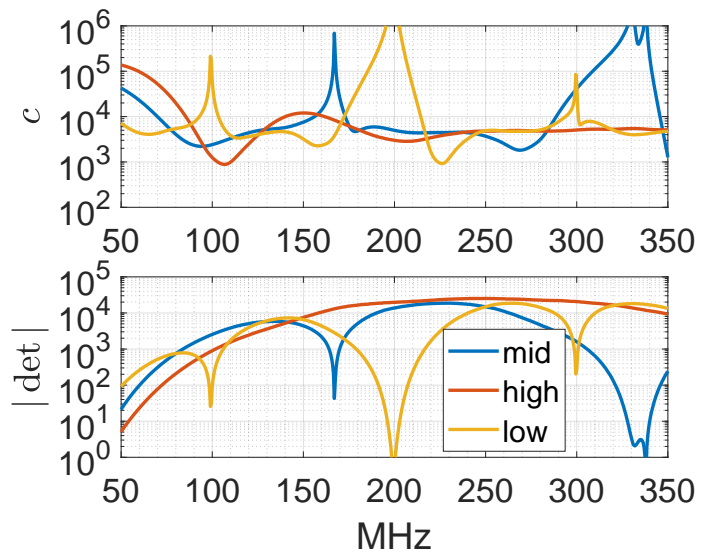

Figure 14. Determinants and conditions numbers of using $A_{Y}$ for the 3 bands: low, mid, high.

and the mean over 100 measurements. We discover strong $\Delta \Gamma$ correlation over frequency as well as the presence of systematic outliers.
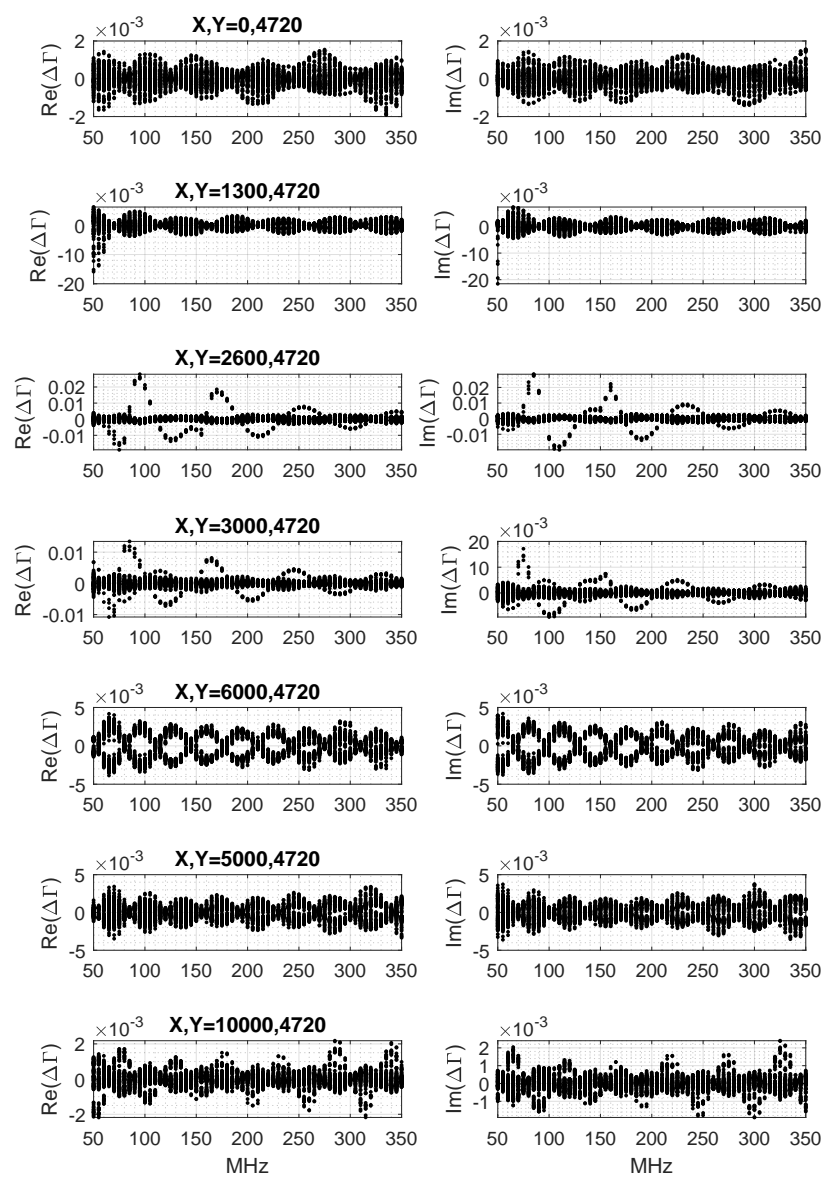

Figure 15. Measured $\Delta \Gamma$ relative to the mean $\Gamma$ of the CCMT-101 tuner at the prescribed tuner positions repeated 100 times.

We use the $\Delta \Gamma$ data to perturb the entries of the $Y_{2,3,4}$ in $\left.A_{Y}\right|_{\gamma_{1}=0}$ and $\Gamma_{2,3,4}$ in $\left.A_{\Gamma_{m o d}}\right|_{\gamma_{1}=0}$ to obtain 100 solutions. 
For each trial we compute

$$
\|\Delta \mathbf{x}\|=\sqrt{\Delta a^{2}+\Delta b^{2}+\Delta c^{2}+\Delta d^{2}}
$$

where the $\Delta$ is taken with respect to the unperturbed solution. The root-mean-square (rms) error is taken as

$$
\|\Delta \mathbf{x}\|_{\mathrm{RMS}}=\sqrt{\frac{1}{100} \sum_{i=1}^{100}\|\Delta \mathbf{x}\|_{i}^{2}}
$$

The results are shown in Fig. 16 relative to the unperturbed solution $\|\mathbf{x}\|$. The results are shown in Fig. 16(a) for $\left.A_{\Gamma_{\text {mod }}}\right|_{\gamma_{1}=0}$ and Fig. 16(b) for $\left.A_{Y}\right|_{\gamma_{1}=0}$. We note that the best case relative errors using $\left.A_{\Gamma_{\text {mod }}}\right|_{\gamma_{1}=0}$ hover at about $10^{-3}$ vs. $10^{-2}$ for $\left.A_{Y}\right|_{\gamma_{1}=0}$. The matrix $\left.A_{\Gamma_{\text {mod }}}\right|_{\gamma_{1}=0}$ again returns consistent solution selection using min. $c$ and max. $\mid$ det $\mid$ all of which are below $10^{-2}$ in relative error. As for $\left.A_{Y}\right|_{\gamma_{1}=0}$, if we assume max. $|\operatorname{det}|$ as the more reliable indicator of solution stability, this would result in the selection of the worst result at $160 \mathrm{MHz}$ with nearly $10 \%$ error. These results suggest that $A_{\Gamma_{\text {mod }}}||_{\gamma_{1}=0}$ is less affected by source matrix error of the type introduced by repeated tuner positioning than the $\left.A_{Y}\right|_{\gamma_{1}=0}$ matrix. Furthermore, the min. $c$ and max. $\mid$ det $\mid$ criteria are equally valid and remain consistent for $\left.A_{\Gamma_{m o d}}\right|_{\gamma_{1}=0}$ in the presence of this type of error. It is also important to note that the low band tuner setting produces highly repeatable solutions at $50 \mathrm{MHz}$ despite being significantly below the minimum tuner rating of $100 \mathrm{MHz}$.

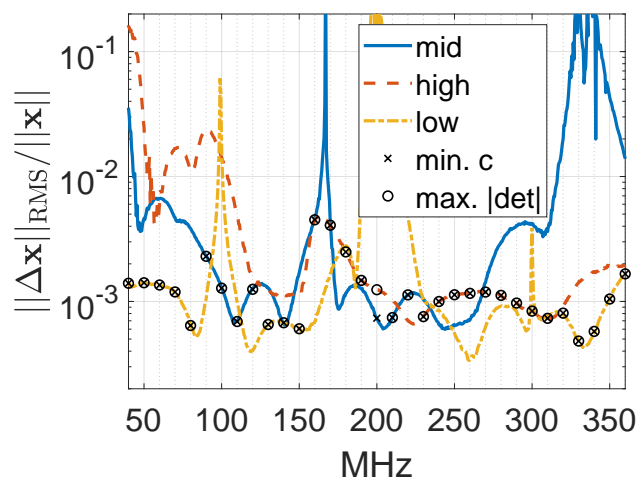

(a)

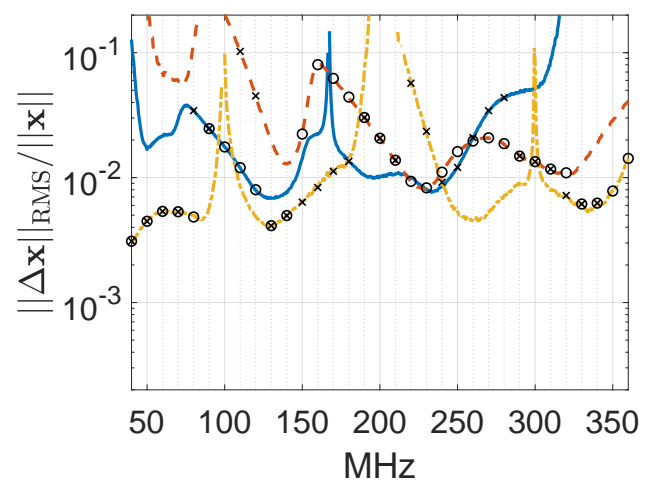

(b)

Figure 16. The error of the solutions due to over repeated tuner positions relative to the unperturbed solution: (a) $\left.A_{\Gamma_{\text {mod }}}\right|_{\gamma_{1}=0}$; (b) $\left.A_{Y}\right|_{\gamma_{1}=0}$

\section{CONCLUSION}

The noise temperature equation based on noise-wave parameters with singularity removed produces strong convergence between source matrix condition number and the matrix determinant for the $4 \times 4$ case. The resulting condition number reaches a minimum at approximately 2.5 while the $|\operatorname{det}|$ is simultaneously maximized at 2.5 for the maximum possible spread of points over the Smith chart given that one point is $|\Gamma|=0$. This provides a solid physical and mathematical basis for quantifying "well-spread" source points for noise parameter extraction. We have derived a simple formula for | det | which is useful for selecting a mismatch device for noise measurement.

We have also shown that the incident power equivalent noise temperature quantity which emerges due to the singularity removal is readily obtained through cold-source measurement and it is also obtainable with hot/cold measurement. We apply the cold source method to noise parameter extraction of a DUT in the SKA-Low band of 50 to $350 \mathrm{MHz}$ using a slide screw tuner rated for $100 \mathrm{MHz}$ to $1 \mathrm{GHz}$ with 7 tuner positions. We obtain repeatable noise parameter solutions in 50 to $100 \mathrm{MHz}$ range below the tuner rating with relative error comparable to that of the 100 to $350 \mathrm{MHz}$ range. Furthermore, the source matrix based on noise-wave parameters with singularity removed produces much clearer prediction of the expected quality of noise parameter extraction than the admittance-based source matrix. The noise-wave based matrix is also less affected by source matrix error due to tuner uncertainties. The method we introduce here, i.e. the condition number of the source matrix and the measurement equation, is readily applicable to leastsquares based measurements.

\section{REFERENCES}

[1] S. J. Tingay et al., "The Murchison Widefield Array: The Square Kilometre Array Precursor at Low Radio Frequencies," Publications of the Astron. Soc. of Australia, vol. 30, p. 7, Jan. 2013.

[2] M. P. van Haarlem et al., "LOFAR: The LOw-Frequency ARray," Astronomy and Astrophysics, vol. 556, p. A2, Aug. 2013.

[3] S. Ellingson, T. Clarke, A. Cohen, J. Craig, N. Kassim, Y. Pihlstrom, L. Rickard, and G. Taylor, "The long wavelength array," Proceedings of the IEEE, vol. 97, no. 8, pp. 1421-1430, Aug 2009.

[4] A. A. Konovalenko, I. S. Falkovich, A. A. Gridin, P. L. Tokarsky, and S. N. Yerin, "UWB active antenna array for low frequency radio astronomy," in 2012 6th International Conference on Ultrawideband and Ultrashort Impulse Signals, Sept 2012, pp. 39-43.

[5] J. G. B. de Vaate et al., "The SKA1_Low telescope: The station design and prototyping," in 2018 2nd URSI Atlantic Radio Science Meeting (AT-RASC), May 2018, pp. 1-1.

[6] M. G. Labate, R. Braun, P. Dewdney, M. Waterson, and J. Wagg, "SKA1LOW: Design and scientific objectives," in 2017 XXXIInd General Assembly and Scientific Symposium of the International Union of Radio Science (URSI GASS), Aug 2017, pp. 1-4.

[7] R. Braun, "SKA1-Low science," in 2018 2nd URSI Atlantic Radio Science Meeting (AT-RASC), May 2018, pp. 1-1.

[8] R. A. Monsalve, A. E. E. Rogers, J. D. Bowman, and T. J. Mozdzen, "Calibration of the EDGES High-band Receiver to Observe the Global $21 \mathrm{~cm}$ Signature from the Epoch of Reionization," The Astrophysical Journal, vol. 835, no. 1, p. 49, Jan 2017.

[9] D. R. DeBoer et al., "Hydrogen Epoch of Reionization Array (HERA)," Publications of the Astronomical Society of the Pacific, vol. 129, no. 974, p. 045001, Apr 2017.

[10] R. Byrne, M. F. Morales, B. Hazelton, W. Li, N. Barry, A. P. Beardsley, R. Joseph, J. Pober, I. Sullivan, and C. Trott, "Fundamental Limitations on the Calibration of Redundant $21 \mathrm{~cm}$ Cosmology Instruments and Implications for HERA and the SKA," The Astrophysical Journal, vol. 875, no. 1, p. 70, Apr 2019. 
[11] L. Belostotski, B. Veidt, K. F. Warnick, and A. Madanayake, "Lownoise amplifier design considerations for use in antenna arrays," IEEE Transactions on Antennas and Propagation, vol. 63, no. 6, pp. 25082520, June 2015.

[12] D. Ung, A. Sutinjo, and D. Davidson, "Evaluating receiver noise temperature of a radio telescope in the presence of mutual coupling: Comparison of current methodologies," in 2019 13th European Conference on Antennas and Propagation (EuCAP), March 2019, pp. 1-3.

[13] R. P. Meys, "A wave approach to the noise properties of linear microwave devices," IEEE Transactions on Microwave Theory and Techniques, vol. 26, no. 1, pp. 34-37, Jan 1978.

[14] R. Hu and S. Weinreb, "A novel wide-band noise-parameter measurement method and its cryogenic application," IEEE Transactions on Microwave Theory and Techniques, vol. 52, no. 5, pp. 1498-1507, May 2004.

[15] R. Q. Lane, "The determination of device noise parameters," Proceedings of the IEEE, vol. 57, no. 8, pp. 1461-1462, Aug 1969.

[16] M. De Dominicis, F. Giannini, E. Limiti, and G. Saggio, "A novel impedance pattern for fast noise measurements," IEEE Transactions on Instrumentation and Measurement, vol. 51, no. 3, pp. 560-564, June 2002.

[17] M. Himmelfarb and L. Belostotski, "On impedance-pattern selection for noise parameter measurement," IEEE Transactions on Microwave Theory and Techniques, vol. 64, no. 1, pp. 258-270, Jan 2016.

[18] S. Van den Bosch and L. Martens, "Improved impedance-pattern generation for automatic noise-parameter determination," IEEE Transactions on Microwave Theory and Techniques, vol. 46, no. 11, pp. 1673-1678, Nov 1998.

[19] M. Sannino, "On the determination of device noise and gain parameters," Proceedings of the IEEE, vol. 67, no. 9, pp. 1364-1366, Sep. 1979.

[20] G. Strang, Introduction to Linear Algebra, 5th ed. Wellesley, MA: Wellesley-Cambridge Press, 2016, ch. 5, 7, 11

[21] A. C. Davidson, B. W. Leake, and E. Strid, "Accuracy improvements in microwave noise parameter measurements," IEEE Transactions on Microwave Theory and Techniques, vol. 37, no. 12, pp. 1973-1978, Dec 1989.

[22] H. V. Nguyen, N. Misljenovic, and B. Hosein, "Efficient noise extraction algorithm and wideband noise measurement system from $0.3 \mathrm{GHz}$ to 67 GHz," in 81st ARFTG Microwave Measurement Conference, June 2013 , pp. 1-2.

[23] A. T. Sutinjo and L. Belostotski, "Analytical determinant of the noise parameter extraction matrix and its applications," in 2019 USNC-URSI Radio Science Meeting (Joint with AP-S Symposium), July 2019.

[24] S. W. Wedge and D. B. Rutledge, "Wave techniques for noise modeling and measurement," IEEE Transactions on Microwave Theory and Techniques, vol. 40, no. 11, pp. 2004-2012, Nov 1992.

[25] A. E. E. Rogers and J. D. Bowman, "Absolute calibration of a wideband antenna and spectrometer for accurate sky noise temperature measurements," Radio Science, vol. 47, no. 6, 2012. [Online]. Available: https://agupubs.onlinelibrary.wiley.com/doi/abs/10.1029/2011RS004962

[26] M. Sokolowski et al., "BIGHORNS-broadband instrument for global HydrOgen ReioNisation signal," Publications of the Astronomical Society of Australia, vol. 32, 2015

[27] A. T. Sutinjo, D. C. X. Ung, and B. Juswardy, "Cold-source noise measurement of a differential input single-ended output low-noise amplifier connected to a low-frequency radio astronomy antenna," IEEE Transactions on Antennas and Propagation, vol. 66, no. 10, pp. 55115520 , Oct 2018

[28] P. Penfield, "Wave representation of amplifier noise," IRE Transactions on Circuit Theory, vol. 9, no. 1, pp. 84-86, March 1962.

[29] V. D. Larock and R. P. Meys, "Automatic noise temperature measurement through frequency variation," IEEE Transactions on Microwave Theory and Techniques, vol. 30, no. 8, pp. 1286-1288, Aug 1982.

[30] R. P. Meys and F. Boukerroum, "Broadband noise system allows measurements according to both standard methods," IEEE Transactions on Instrumentation and Measurement, vol. 60, no. 4, pp. 1316-1327, April 2011.

[31] L. Belostotski and J. W. Haslett, "Evaluation of tuner-based noiseparameter extraction methods for very low noise amplifiers," IEEE Transactions on Microwave Theory and Techniques, vol. 58, no. 1, pp. 236-250, Jan 2010.

[32] "How does a slide screw tuner (frequency $>100 \mathrm{MHz}$ ) work?" Focus Microwaves. [Online]. Available: https://focus-microwaves.com/wpcontent/uploads/2017/02/How-does-a-Slide-Screw-Tuner-work.pdf

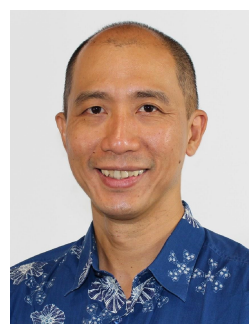

Adrian T. Sutinjo (SM'15) received the B.S.E.E. degree from Iowa State University, Ames, IA, USA, in 1995, the M.S.E.E. degree from the Missouri University of Science and Technology, Rolla, MO, USA, in 1997, and the Ph.D. degree in electrical engineering from the University of Calgary, Calgary, AB, Canada, in 2009. From 1997 to 2004, he was an RF Engineer with Motorola, Schaumburg, IL, USA, and with Murandi Communications Ltd., Calgary. $\mathrm{He}$ is currently a Senior Lecturer with the School of Electrical Engineering, Computing and Mathematics, Curtin University, Perth, WA, Australia. He has been with the International Centre for Radio Astronomy Research/Curtin Institute of Radio Astronomy, Curtin University, Bentley, WA, Australia, since 2012. His current research interests include antennas, $\mathrm{RF}$ and microwave engineering, electromagnetics, and radio astronomy engineering.

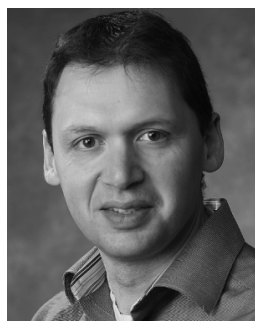

Leonid Belostotski (S'97-M'01-SM'14) Leonid Belostotski received the B.Sc. and M.Sc. degrees in electrical engineering from the University of Alberta, Edmonton, AB, Canada, in 1997 and 2000, respectively, and the Ph.D. degree from the University of Calgary, Calgary, AB, Canada, in 2007. He was an RF Engineer with Murandi Communications Ltd., Calgary, from 2001 to 2004 . He is currently a Professor with the University of Calgary and the Canada Research Chair in high-sensitivity radiometers and receivers. His current research interests include RF and mixed-signal ICs, high-sensitivity receiver systems, antenna arrays, and terahertz systems. He was a recipient of the IEEE Microwave Theory and Techniques-11 Contest on Creativity and Originality in Microwave Measurements, in 2008, and the Outstanding Student Designer Award from Analog Devices, Inc., in 2007. He is the IEEE Southern Alberta Solid-State, Circuits and Circuits and Systems Chapter's Chair. He is an Associate Editor of the IEEE Transactions on Instrumentation and Measurement.

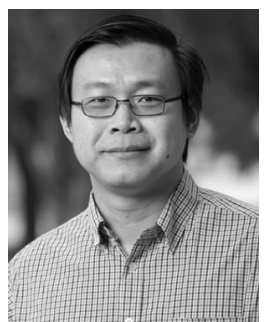

Budi Juswardy (S'99-M'10) received the B.Eng. degree in electrical engineering from the National University of Singapore, Singapore, in 2002, the M.Sc. degree from Nanyang Technological University, Singapore, in 2005, and the Ph.D. degree from Edith Cowan University, Joondalup, WA, Australia, in 2010, respectively. From 2001 to 2002, he was with the Institute of Microelectronic, Singapore, where he was involved in a CMOS SRAM and deltasigma modulator. He was an Electronics Engineer with Motorola Electronics Pte Ltd., Singapore, from 2003 to 2006. He was with the Western Australian Centre for Microphotonic Systems, Edith Cowan University, Joondalup, WA, Australia, where he was involved in optical signal processing until 2010 . He is currently with the Curtin Institute of Radio Astronomy, Curtin University, Bentley, WA, Australia, where he has been involved in the Square Kilometre Array Project. His current research interests include RF circuit design and analysis, optical communication, phased-array antennas, radio astronomy instrumentation, electromagnetic interference emission measurements, and low-emission power supply systems. 


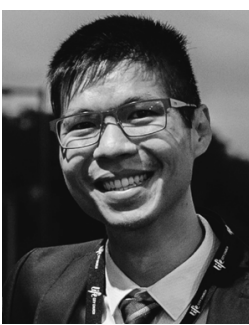

Daniel C. X. Ung (GS'16) received the B.Eng. degree in E.C. from Curtin University, Perth, WA, Australia, in 2015, where he is currently pursuing the part-time M.Phil. degree.,He has been a Support Engineer with the International Centre for Radio Astronomy Research/Curtin Institute of Radio Astronomy, Curtin University, Bentley, WA, Australia, since 2015.,Mr. Ung received the first place in the FEKO Student Competition hosted by Altair Engineering in 2016. His winning entry-Embedded Element Pattern Beam Model for Murchison Widefield Array-enabled an accurate and accessible beam pattern of the Murchison Widefield Array for astronomers. He received a summer studentship at the International Centre for Radio Astronomy Research in 2014. 